\title{
Adaptive Integral Method for Higher Order Method of Moments
}

\author{
Kim, Oleksiy S.; Meincke, Peter
}

Published in:

I E E E Transactions on Antennas and Propagation

Link to article, DOI:

10.1109/TAP.2008.926759

Publication date:

2008

Document Version

Publisher's PDF, also known as Version of record

Link back to DTU Orbit

Citation (APA):

Kim, O. S., \& Meincke, P. (2008). Adaptive Integral Method for Higher Order Method of Moments. I E E E Transactions on Antennas and Propagation, 56(8), 2298-2305. https://doi.org/10.1109/TAP.2008.926759

\section{General rights}

Copyright and moral rights for the publications made accessible in the public portal are retained by the authors and/or other copyright owners and it is a condition of accessing publications that users recognise and abide by the legal requirements associated with these rights.

- Users may download and print one copy of any publication from the public portal for the purpose of private study or research.

- You may not further distribute the material or use it for any profit-making activity or commercial gain

- You may freely distribute the URL identifying the publication in the public portal

If you believe that this document breaches copyright please contact us providing details, and we will remove access to the work immediately and investigate your claim. 


\title{
Adaptive Integral Method for Higher Order Method of Moments
}

\author{
Oleksiy S. Kim and Peter Meincke, Member, IEEE
}

\begin{abstract}
The adaptive integral method (AIM) is combined with the higher order method of moments (MoM) to solve integral equations. The technique takes advantage of the low computational complexity and memory requirements of the AIM and the reduced number of unknowns and higher order convergence of higher order basis functions. The classical AIM is appropriately modified to allow larger discretization elements and, consequently, higher basis function expansion orders. Numerical examples based on the higher order hierarchical Legendre basis functions show the advantages of the proposed technique over the classical AIM based on low-order basis functions in terms of memory and computational time.
\end{abstract}

Index Terms-Adaptive integral method (AIM), higher order hierarchical Legendre basis functions, integral equations, method of moments (MoM), scattering.

\section{INTRODUCTION}

$\mathbf{T}$ HE METHOD of moments (MoM) has been widely used in the past decades to solve electromagnetic scattering problems formulated in terms of integral equations [1]-[3]. The conventional straightforward application of MoM involves low-order basis functions with support in mesh cells with size in the order of one tenth of a wavelength, leading to a dense system of linear equations. The resulting memory requirement is $O\left(N^{2}\right)$ and the solution complexity $O\left(N^{3}\right)$ for a direct solver and $O\left(N^{2}\right)$ for an iterative one, with $N$ being the number of unknowns. Hence, the computational demands increase drastically as the problem size grows.

Several techniques have been proposed to reduce the memory demands as well as the solution complexity of the conventional MoM. Fast integral equation solvers, such as the multilevel fast multiple method (MLFMM) [4], [5], the adaptive integral method (AIM) [6], [7] and its close counterpart the precorrected FFT (PC-FFT) [8], [9] reach the solution complexity $O(N \log N)$. Furthermore, MLFMM and AIM are able to reduce the memory demands for volumetric problems to $O(N \log N)$ and $O(N)$, respectively, and to $O(N \log N)$ and $O\left(N^{1.5}\right)$, respectively, for surface problems [10]. Higher order basis functions can also be employed in the conventional MoM to significantly reduce the number of unknowns, which in many practical cases is more memory and computationally efficient as compared to the fast solvers based on low-order basis functions [11]. An obvious advantage is obtained by combining the fast

Manuscript received June 29, 2007; revised April 7, 2008. Published August 6, 2008 (projected).

The authors are with the Department of Electrical Engineering, ElectroScience Section, Technical University of Denmark, DK-2800 Kgs. Lyngby, Denmark (e-mail: osk@elektro.dtu.dk; pme@elektro.dtu.dk).

Digital Object Identifier 10.1109/TAP.2008.926759 solvers with higher order basis functions. This approach has been pursued in [12] and [13], in which MLFMM is applied to surface integral equations (SIE) discretized with higher order interpolatory basis functions. The computational complexity and memory requirements of this technique are addressed in [14]. The PC-FFT technique along with the higher order locally corrected Nyström method are used in [15] to solve SIE for metallic objects.

In this paper, AIM is employed to accelerate the higher order MoM solution of volume and volume-surface integral equations. Among various types of higher order basis functions a set of hierarchical basis functions based on the orthogonal Legendre polynomials are chosen [16] due to their favorable properties with respect to the iterative solver convergence [17]. As compared to the basis functions utilized in [15], the higher order Legendre basis functions maintain continuity of the unknown function across a boundary between discretization elements, which improves accuracy and convergence of the solution.

The remaining of this paper is organized as follows. In Section II the procedure for solving integral equations using higher order orthogonal Legendre polynomials is briefly reviewed. The application of the higher order basis functions becomes advantageous when they are defined on relatively large subdomains. However, this large subdomain size contradicts with the accuracy conditions for multipole expansions established within the framework of AIM [6]. The appropriate modifications are developed in Section III, so that the low computational complexity and memory requirements of AIM are preserved, even when combined with higher order basis functions. In Section IV numerical examples are given to validate the presented technique as well as to show its efficiency. The examples involve solutions of volume, surface and volume-surface integral equations. Finally, the conclusions are given in Section V.

\section{MoM}

The MoM is a discretization scheme for equations of the form

$$
\mathcal{L} \mathbf{f}=\mathrm{g}
$$

where $\mathcal{L}$ is a linear integro-differential operator, $\mathrm{g}$ is a known function, and $\mathbf{f}$ is the unknown function to be determined. The first step in the MoM solution is to approximate the unknown function $\mathbf{f}$ by a linear combination of known basis functions $\mathbf{B}_{n}$, $n=1,2, \ldots, N$ as

$$
\mathbf{f}=\sum_{n=1}^{N} I_{n} \mathbf{B}_{n}
$$


where $I_{n}$ are unknown coefficients. Inserting (2) into (1) and testing with a set of known functions $\mathbf{T}_{m}$ yields the matrix equation

$$
\overline{\mathbf{Z}} \mathbf{I}=\mathbf{V} \text {. }
$$

The MoM matrix $\overline{\mathbf{Z}}$ and the excitation vector $\mathbf{V}$ have the elements

$$
Z_{m n}=\left\langle\mathbf{T}_{m}, \mathcal{L} \mathbf{B}_{n}\right\rangle
$$

and

$$
V_{m}=\left\langle\mathbf{T}_{m}, \mathbf{g}\right\rangle
$$

with $\langle\cdot, \cdot\rangle$ denoting the inner product. For subdomain basis functions, for which the AIM is applicable, the discretization procedure (2) includes representation of the object of interest by smaller regular geometrical elements (such as triangles, quadrilaterals, hexahedra, etc.) and the basis functions are then defined on these elements, also referred to as subdomains. To improve the convergence of the solution the continuity condition is usually imposed for either tangential or normal components of the basis functions at the boundaries between subdomains. Thus, a basis function can span more than one subdomain. The rooftop basis function is an example of a function defined on two neighbor elements, and it belongs to the class of low-order basis functions, i.e., functions defined by zero or first-order polynomials. Higher order basis functions involve second, third, or higher polynomial expansion orders.

In this work, the contravariant components $f^{\xi}$ of the unknown function $\mathbf{f}$ are represented in terms of the higher order hierarchical Legendre basis functions [16] as

$$
f^{\xi}=\frac{1}{\mathcal{J}} \sum_{m=0}^{M^{\xi}} \sum_{n=0}^{N^{\eta}} \sum_{q=0}^{Q^{\zeta}} I_{m n q}^{\xi} P_{m}^{\prime}(\xi) P_{n}(\eta) P_{q}(\zeta)
$$

in volume hexahedral elements and as

$$
f^{\xi}=\frac{1}{\mathcal{J}_{S}} \sum_{m=0}^{M^{\xi}} \sum_{n=0}^{N^{\eta}} I_{m n}^{\xi} P_{m}^{\prime}(\xi) P_{n}(\eta)
$$

on surface quadrilateral elements. In (5) and (6), $\mathcal{J}$ and $\mathcal{J}_{S}$ are the Jacobians of the parametric transformation between the local curvilinear coordinate system of the subdomain and the physical space coordinates for hexahedra and quadrilaterals, respectively,

$$
P_{m}^{\prime}(\xi)= \begin{cases}1-\xi, & m=0 \\ 1+\xi, & m=1 \\ P_{m}(\xi)-P_{m-2}(\xi), & m \geq 2\end{cases}
$$

$P_{m}(\xi)$ are Legendre polynomials, and $M^{\xi}, N^{\zeta}$, and $Q^{\eta}$ denote the expansion orders along the parametric directions.

The expansions (5) and (6) include the subset of first and higher order rooftop functions with indices $m=0$ and 1 . These rooftop functions ensure continuity of the normal component of the unknown function across the boundary between two neighbor subdomains. Thus, the rooftop functions span two subdomains while the remaining higher order functions are defined on a single subdomain.

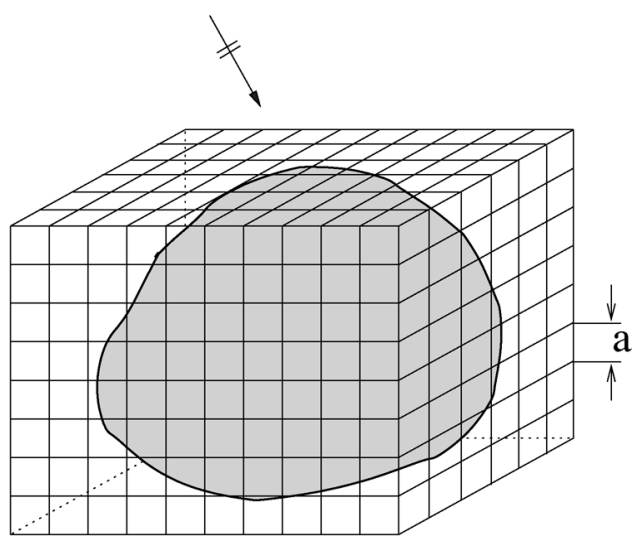

Fig. 1. Auxiliary Cartesian grid for the far-field interaction computations in AIM.

\section{Formulation OF THE AIM FOR HighER ORDER MOM}

The AIM accelerates the solution of an integral equation by separating elements of the MoM matrix, responsible for nearand far-field interactions, as $\overline{\mathbf{Z}}=\overline{\mathbf{Z}}^{\text {near }}+\overline{\mathbf{Z}}^{\text {far }}$. The near-field elements are defined as

$$
z_{m n}^{\text {near }}= \begin{cases}z_{m n}-z_{m n}^{\mathrm{far}} & d_{m n}<d_{\text {near }} \\ 0, & \text { otherwise }\end{cases}
$$

where $d_{m n}$ is the distance between the centers of the corresponding basis function support subdomains, and $d_{\text {near }}$ is the near-field range. The near-field elements, which constitute a relatively small part of the full matrix, are computed explicitly and stored in a sparse-matrix format. The far-field interactions are accounted for implicitly in the matrix-vector product in each iteration of the iterative solution of the MoM matrix system. Here, to apply AIM, the components $f_{\alpha} \in\left\{f_{x}, f_{y}, f_{z}, \nabla \cdot \mathbf{f}\right\}$ of the basis functions $\mathbf{f}(\mathbf{r})$ are expanded in terms of Dirac delta functions defined at nodes of a regular Cartesian grid enclosing the object (see Fig. 1) as

$$
f_{\alpha}(\mathbf{r}) \simeq \sum_{\mathbf{u} \in C_{\alpha}} \Lambda_{\alpha \mathbf{u}} \delta(\mathbf{r}-\mathbf{u})
$$

where $C_{\alpha}$ is a cube of $(L+1)^{3}$ nodes. The coefficients $\Lambda_{\alpha \mathbf{u}}$ are chosen so that the expansion reproduces the far field radiated by $f_{\alpha}$. Subsequently, the computation of the matrix-vector product $\overline{\mathbf{Z}}^{\mathrm{far}} \mathbf{I}$ at each iteration of the iterative solution process is sped up by application of the FFT. The accuracy of the expansion in (9) is controlled by the multipole expansion order $L$, the support size $b$ of the expanded function, and the grid step size $a$. To assure a reasonable error, both $k_{0} a$ and $k_{0} b$ should satisfy the relation [6]

$$
\frac{\left(\max \{b, L a\} k_{0}\right)^{L+1}}{(L+1) !} \ll 1
$$

where $k_{0}$ is the wavenumber in free space. In other words, the AIM works fine for low-order basis functions since their support size $b$ is relatively small. However, the higher order basis functions become advantageous when they are defined on relatively large subdomains, allowing high expansion orders. Obviously, this contradicts the accuracy condition (10). This is the case especially for basis functions that span two or more subdomains, 


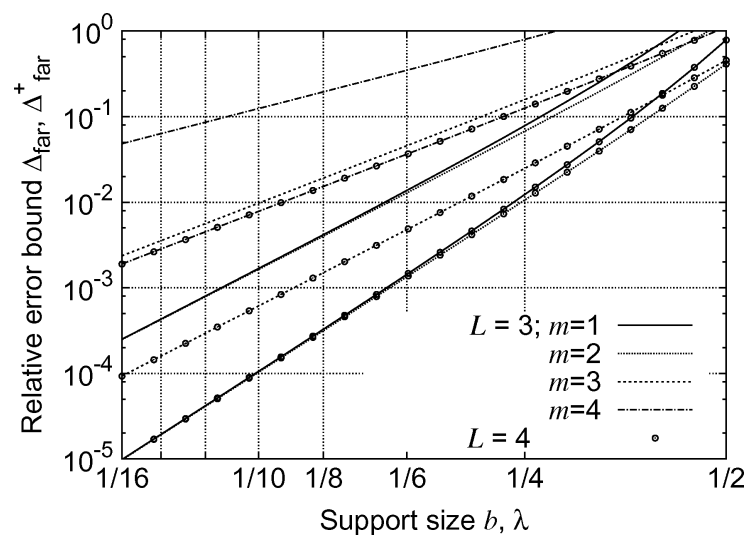

Fig. 2. Relative error bound as a function of the support size $b$.

for which the support size $b$ is the sum of the corresponding element sizes.

To alleviate this contradiction it is here proposed to expand the parts of the basis functions, which are defined on several subdomains, separately in each subdomain. Thus, the support size $b$ is effectively reduced to the size of a single subdomain $h$, implying that larger discretization elements with higher basis function expansion orders can be utilized. For instance, if the rooftop basis function is expressed as a sum of two subfunctions defined on each subdomain as $f_{\alpha}(\mathbf{r})=f_{\alpha}^{+}(\mathbf{r})+f_{\alpha}^{-}(\mathbf{r})$, (9) can be modified as

$$
\begin{aligned}
f_{\alpha}(\mathbf{r}) & =f_{\alpha}^{+}(\mathbf{r})+f_{\alpha}^{-}(\mathbf{r}) \\
& \simeq \sum_{\mathbf{u} \in C_{\alpha}^{+}} \Lambda_{\alpha \mathbf{u}}^{+} \delta(\mathbf{r}-\mathbf{u})+\sum_{\mathbf{u} \in C_{\alpha}^{-}} \Lambda_{\alpha \mathbf{u}}^{-} \delta(\mathbf{r}-\mathbf{u})
\end{aligned}
$$

This expansion requires two matrices $\Lambda_{\alpha \mathbf{u}}^{+}$and $\Lambda_{\alpha \mathbf{u}}^{-}$to store the coefficients. One of them is also used store the coefficients for basis functions defined on a single subdomain.

As it is shown in [6], the relative far-field error bound $\Delta_{\text {far }}$ of the expansion (9) satisfies

$$
\Delta_{\text {far }} \leq 2 \max _{\alpha, \mathbf{k}} \Delta \tilde{f}_{\alpha}(\mathbf{k})=2 \max _{\alpha, \mathbf{k}} \frac{\left|\delta \tilde{f}_{\alpha}(\mathbf{k})\right|}{\left|\tilde{f}_{\alpha}(\mathbf{k})\right|}
$$

where $\tilde{f}_{\alpha}(\mathbf{k})$ is the Fourier transform of $f_{\alpha}(\mathbf{r}), \mathbf{k}=k_{0} \hat{\mathbf{r}}$, and

$$
\begin{aligned}
\left|\delta \tilde{f}_{\alpha}(\mathbf{k})\right| \leq & \frac{2}{(L+1) !}\left(\frac{b k_{0}}{2}\right)^{L+1} \\
& \times \int\left|f_{\alpha}(\mathbf{r})\right| d \mathbf{r}\left[1+O\left(b k_{0}\right)\right] \\
\text { for } L \geq & 1,0 \leq a \leq 2 b / \max \{5, L\} .
\end{aligned}
$$

For the modified expansion (11) it can be shown that

$$
\begin{aligned}
\Delta_{\text {far }} & \leq 2 \max _{\alpha, \mathbf{k}}\left\{\frac{2\left|\delta \tilde{f}_{\alpha}^{+}(\mathbf{k})\right|}{\left|\tilde{f}_{\alpha}(\mathbf{k})\right|}, \frac{2\left|\delta \tilde{f}_{\alpha}^{-}(\mathbf{k})\right|}{\left|\tilde{f}_{\alpha}(\mathbf{k})\right|}\right\} \\
& =\max _{\alpha, \mathbf{k}}\left\{\Delta_{\text {far }}^{+}, \Delta_{\text {far }}^{-}\right\}
\end{aligned}
$$

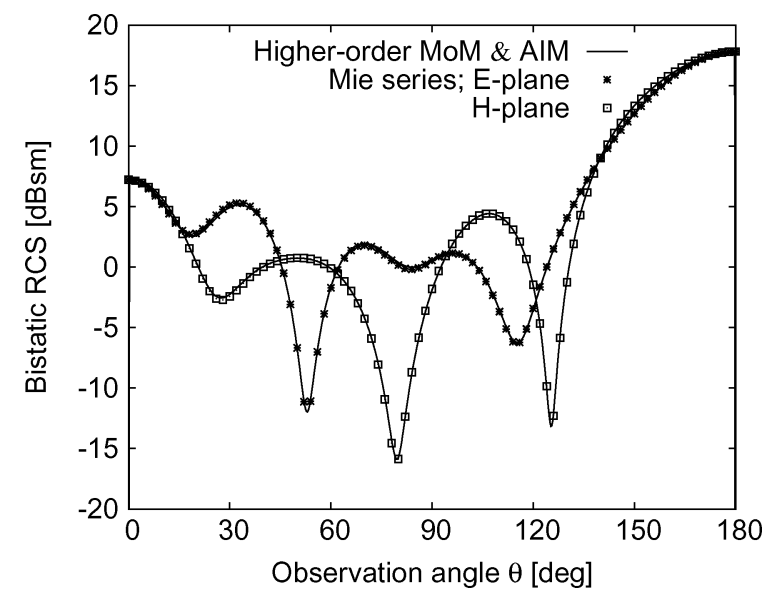

Fig. 3. Bistatic RCS of a $1.5 \lambda_{0}$ dielectric sphere with $\varepsilon_{r}=4.0$.

where $\left|\delta \tilde{f}_{\alpha}^{+}(\mathbf{k})\right|$ and $\left|\delta \tilde{f}_{\alpha}^{-}(\mathbf{k})\right|$ are estimated separately for each subdomain using (13) with $b=h$. For a symmetric basis function defined on equal subdomains it is sufficient to perform this estimation for only one of them.

To illustrate the effect of the modification in (11) on the error $\Delta_{\text {far }}$ consider a one dimensional rooftop basis function, which is defined on two equal subdomains as

$$
f_{1}(x)= \begin{cases}1+x / h, & -h \leq x<0 \\ 1-x / h, & 0 \leq x \leq h \\ 0, & \text { otherwise }\end{cases}
$$

and a function, which is defined on a single subdomain as $f_{2}(x)=P_{m}^{\prime}(2 x / h),-h / 2 \leq x \leq h / 2$. The first function has the support size $b=2 h$. The second function represents one half of the rooftop basis function (say $f_{\alpha}^{+}$) if $m=1$, or a higher order basis function if $m \geq 2$, and its support size is $b=h$. Fig. 2 shows the relative error bound $\left.\Delta_{\text {far }}^{+}\right|_{\mathbf{k}=k_{0} \hat{x}}$ for $f_{2}(x)$ as a function of the support size $b$. The order $m$ is varied from $m=1$ to $m=4$ for the multipole expansion orders $L=3$ and $L=4$. It is easily seen, that $\Delta_{\text {far }}$ estimated for $f_{1}(x)$ according to (12) is equal to $\Delta_{\text {far }}^{+}$estimated for $\left.f_{2}(x)\right|_{m=1}$ according to (14).

For the function $f_{1}(x)$ with $h=\lambda_{0} / 8, \lambda_{0}$ being the wavelength in free space, $b=2 h=\lambda_{0} / 4$, and the multipole expansion order $L=4$ yields $\Delta_{\text {far }} \leq 0.012$. When we apply our modified expansion (11) to the same function, the error bound decreases to $\Delta_{\text {far }}=\Delta_{\text {far }}^{+} \leq 0.0003$ for $L=4$, since now $b=$ $h=\lambda_{0} / 8$. Thus, we can undoubtedly decrease the multipole expansion order to $L=3$ and achieve the error $\Delta_{\text {far }} \leq 0.004$, which is still much lower than the initial bound $\Delta_{\text {far }} \leq 0.012$. However, this occurs at the expense of a finer AIM auxiliary grid, since the grid step size $a$ decreases with $b$ according to (13b).

Alternatively, we can keep $L=4$, increase the subdomain size to $h=b=\lambda_{0} / 4$ and use the higher basis functions with orders up to $m=2$. In this case, the AIM auxiliary grid remains unchanged and the number of unknowns is also nearly the same, but the accuracy of the final solution increases. Notably, the error curve corresponding to $m=2$ in Fig. 2 is even slightly lower than the curve for $m=1$, which ensures that the 
TABLE I

MoM ANd AIM Parameters USEd for SOlving the SCATTERING Problem For a $1.5 \lambda_{0}$ Dielectric SPHERE

\begin{tabular}{|c|c|c|c|c|c|c|c|c|c|}
\hline $\begin{array}{l}\text { Case } \\
\text { no. }\end{array}$ & Grid size & $a, \lambda_{0}$ & $b^{*}, \lambda_{0}$ & $L$ & $d_{\text {near, }}, \lambda_{0}$ & Memory, MB & $\begin{array}{l}\text { Seconds per } \\
\text { iteration** }\end{array}$ & $\begin{array}{l}\overline{\mathbf{Z}}^{\text {near }} \text { filling } \\
\text { time**, h }^{*}\end{array}$ & $\begin{array}{l}\text { RMS error, } \\
\text { dB }\end{array}$ \\
\hline
\end{tabular}

Mesh: 2048 elements; $h^{*}=1.02 \cdot 10^{-1} \lambda_{0} ; M^{\xi}=2 ; 49,920$ unknowns; modified expansion (11) is applied

\begin{tabular}{|c|c|c|c|c|c|c|c|c|c|}
\hline & \\
\hline 1 & $60 \times 60 \times 60$ & $3.25 \cdot 10^{-2}$ & $1.02 \cdot 10^{-1}$ & $3 \ldots 4$ & 0.160 & 1042 & 3.7 & 1.88 & 0.11 \\
\hline 2 & $54 \times 54 \times 54$ & $3.66 \cdot 10^{-2}$ & $1.02 \cdot 10^{-1}$ & $3 \ldots 4$ & 0.170 & 1396 & 3.3 & 2.19 & 0.11 \\
\hline 3 & $48 \times 48 \times 48$ & $4.06 \cdot 10^{-2}$ & $1.02 \cdot 10^{-1}$ & $3 \ldots 4$ & 0.180 & 1564 & 3.0 & 2.41 & 0.11 \\
\hline 4 & $45 \times 45 \times 45$ & $4.47 \cdot 10^{-2}$ & $1.02 \cdot 10^{-1}$ & $3 \ldots 4$ & 0.183 & 1613 & 2.8 & 2.43 & 0.11 \\
\hline 5 & $40 \times 40 \times 40$ & $4.87 \cdot 10^{-2}$ & $1.02 \cdot 10^{-1}$ & $3 \ldots 4$ & 0.188 & 1692 & 2.5 & 2.51 & 0.11 \\
\hline \multicolumn{10}{|c|}{ Mesh: 256 elements; $h^{*}=2.17 \cdot 10^{-1} \lambda_{0} ; M^{\xi}=3 ; 21,168$ unknowns; modified expansion (11) is applied } \\
\hline 6 & $30 \times 30 \times 30$ & $9.07 \cdot 10^{-2}$ & $2.17 \cdot 10^{-1}$ & $4 \ldots 5$ & 0.350 & 1547 & 1.3 & 3.35 & 0.11 \\
\hline \multicolumn{10}{|c|}{ Mesh: 17051 elements; $h^{*}=4.82 \cdot 10^{-2} \lambda_{0} ; M^{\xi}=1 ; 52,020$ unknowns; expansion (9) is applied } \\
\hline 7 & $60 \times 60 \times 60$ & $3.25 \cdot 10^{-2}$ & $9.48 \cdot 10^{-2}$ & $2 \ldots 4$ & 0.160 & 1201 & 3.8 & 15.33 & 0.65 \\
\hline 8 & $48 \times 48 \times 48$ & $4.06 \cdot 10^{-2}$ & $9.48 \cdot 10^{-2}$ & $2 \ldots 4$ & 0.180 & 1590 & 2.7 & 18.87 & 0.65 \\
\hline 9 & $40 \times 40 \times 40$ & $4.87 \cdot 10^{-2}$ & $9.48 \cdot 10^{-2}$ & $2 \ldots 4$ & 0.188 & 1722 & 2.2 & 20.08 & 0.67 \\
\hline 10 & $40 \times 40 \times 40$ & $4.87 \cdot 10^{-2}$ & $9.48 \cdot 10^{-2}$ & $2 \ldots 4$ & 0.220 & 2640 & 2.5 & 26.60 & 0.67 \\
\hline
\end{tabular}

* average.

** on a 4-CPU $2200 \mathrm{MHz}$ desktop PC.

error bound still satisfies $\Delta^{\text {far }} \leq 0.012$ for $L=4$. With the original expansion (9) this would be nearly impossible because the support size would then be $b=2 h=\lambda_{0} / 2$, and the dramatic error increase could not be prevented by increasing the multipole expansion order $L$.

It is observed that the error bound in Fig. 2 increases as the basis function order $m$ exceeds 2 . Analysis similar to that given above shows that in order to use $m=3$ with the subdomain size $h=b=\lambda_{0} / 3$ and keep the error bound at the level $\Delta^{\mathrm{far}} \leq 0.012$, the multipole expansion order must be increased to $L=5$. In this case, the accuracy of the final solution further enhances and the AIM auxiliary grid becomes coarser. The high error bound for the basis functions with orders $m \geq 4$ makes their usage practically inefficient in the context of AIM. Indeed, for $m=4$ a reasonable error can be achieved with $b<\lambda_{0} / 4$ and $L=5$. However, in this case the problem becomes heavily overdiscretized. Support sizes larger than $b>\lambda_{0} / 4$ require multipole expansion orders higher than $L>5$, for which the numerical procedure for computing the coefficients $\Lambda_{\alpha u}$ is very unstable even with double precision arithmetics. It should also be noted that although the absolute size of the matrix $\overline{\mathbf{Z}}^{\text {near }}$ tends to grow as the expansion order $M^{\xi}$ increases, the complexity of the memory requirement remains unchanged.

The considerations given above, along with the relative error bounds (12) and (14), can be used to determine the multipole expansion orders $L$ individually for each basis function. In our implementation, however, we undertake another approach. We use the same $L$ for all basis functions in a given subdomain based on the error bound corresponding to the basis function with the highest order $m$. Thus, we ensure that the relative error for all basis functions in this subdomain is less than some preset value, and at the same time this requires only one computation of the inverse of the Vandermonde's matrix for determining the coefficients $\Lambda_{\alpha \mathbf{u}}$ in each subdomain. The grid step size $a$ is selected according to (13b) using the average support size $b$ along each dimension. The near-field range $d_{\text {near }}$ should, as a rule of thumb, not be less than $L a$ [6].

\section{NUMERICAL EXAMPLES}

The AIM technique presented in the previous section has been implemented for the volume and volume-surface integral equations solved with the higher order MoM as described in [11] and [18], respectively. In all simulations, the generalized minimal residual (GMRES) iterative algorithm with restarts after 30 iterations has been employed. All calculations are performed with the double precision arithmetic.

In the first example, plane wave scattering by a solid sphere of diameter $1.5 \lambda_{0}$ made of lossless dielectric with relative permittivity $\varepsilon_{r}=4.0$ is considered. The exact Mie series solution serves as a reference. Table I summarizes the AIM parameters for three mesh sizes and expansion orders $M^{\xi}$ used to solve the problem. Cases 1-5 correspond to the mesh composed of 2048 hexahedral elements and the expansion order $M_{\xi}=2$, yielding 49920 unknowns. The AIM procedure uses the modified expansion (11) with the grid step size $a$ varying from $3.25 \cdot 10^{-2} \lambda_{0}$ to $4.87 \cdot 10^{-2} \lambda_{0}$. The near-field range $d_{\text {near }}$ needs to be enlarged as the grid step size $a$ increases so that the resulting root mean square (RMS) error for the bistatic radar cross section (RCS) remains equal to $0.11 \mathrm{~dB}$. Consequently, the memory consumption and the matrix $\overline{\mathbf{Z}}^{\text {near }}$ filling time also grow, while the time spent per iteration decreases due to the coarser AIM auxiliary grid. Thus, the optimal choice of the AIM parameters in each specific case is a compromise between the memory consumption, solution time, and required accuracy.

Next we consider the situation, in which low-order basis functions and the original AIM expansion (9) are used to solve the problem with the same number of unknowns. The scenario is illustrated in Table I in lines 7-10 for a 17 051-element mesh that yields 52020 unknowns with $M^{\xi}=1$. The average support size $b$ is nearly the same as in the previous cases $1-5$. To make a direct comparison possible the AIM auxiliary grid and the near-field range $d_{\text {near }}$ in the cases 7,8 , and 9 are the same as in the cases 1, 3, and 5, respectively. It is observed that the resulting RMS error is relatively high compared to the cases $1-5$ 
TABLE II

AIM PARAMETERS FOR THE SimULATIONS IN Fig. 4

\begin{tabular}{c|c|c|c|c|c|c|c}
\hline Mesh & $M^{\xi}$ & Unknowns & Grid size & $a, \lambda_{0}$ & $b$ (average), $\lambda_{0}$ & $L$ & $d_{\text {near, }} \lambda_{0}$ \\
\hline $22 \times 22 \times 22$ & 1 & 33396 & $64 \times 64 \times 64$ & $2.48 \cdot 10^{-2}$ & $6.36 \cdot 10^{-2}$ & 3 & $1.86 \cdot 10^{-1}$ \\
$12 \times 12 \times 12$ & 2 & 43200 & $40 \times 40 \times 40$ & $4.54 \cdot 10^{-2}$ & $1.17 \cdot 10^{-1}$ & 4 & $2.50 \cdot 10^{-1}$ \\
$8 \times 8 \times 8$ & 3 & 43200 & $30 \times 30 \times 30$ & $6.82 \cdot 10^{-2}$ & $1.75 \cdot 10^{-1}$ & 4 & $4.50 \cdot 10^{-1}$ \\
$6 \times 6 \times 6$ & 4 & 43200 & $30 \times 30 \times 30$ & $9.09 \cdot 10^{-2}$ & $2.33 \cdot 10^{-1}$ & 5 & $5.00 \cdot 10^{-1}$ \\
\hline
\end{tabular}

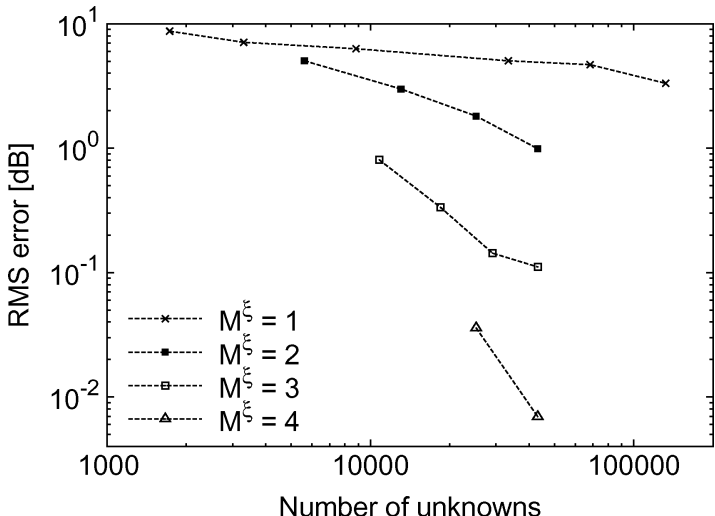

(a)

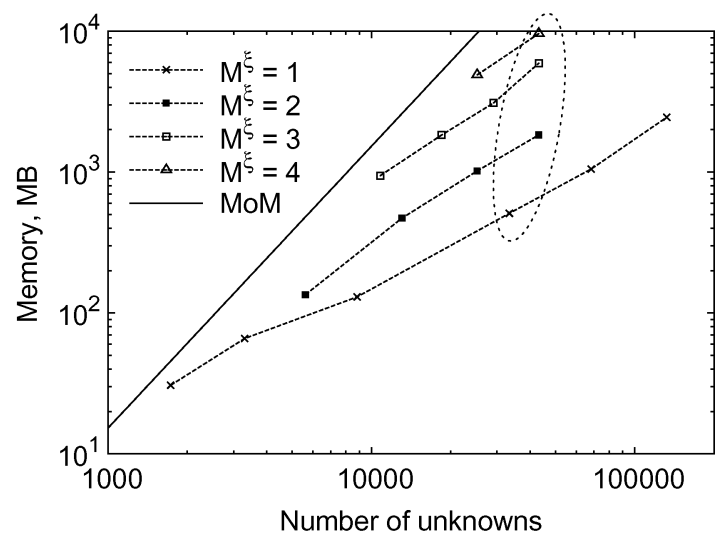

(b)



(c)

Fig. 4. RMS error, the computer memory used, and the time spent at each iteration versus the number of unknowns. The results are for a $1.4 \lambda_{0} \times 1.4 \lambda_{0} \times 1.4 \lambda_{0}$ dielectric cube with $\varepsilon_{r}=4.0$.

where $M^{\xi}=2$, and it is not improved even when $d_{\text {near }}$ is increased (case 10). This illustrates that the limiting factor for the

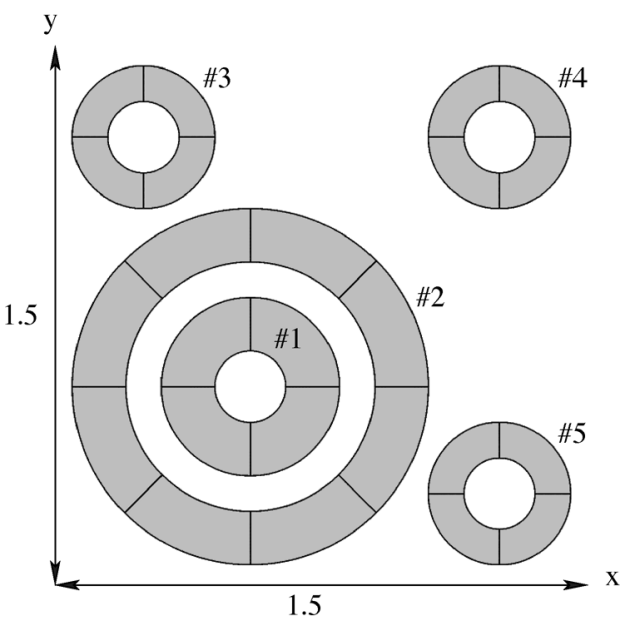

Fig. 5. Unit cell for a $10 \times 10$ array. Parameters of the PEC rings are $\left(x_{c}, y_{c}\right)$, inner, outer radius $\left(r_{i}, r_{o}\right): \# 1\left(0.5 \lambda_{0}, 0.5 \lambda_{0}\right),\left(0.1 \lambda_{0}, 0.25 \lambda_{0}\right)$; $\# 2\left(0.5 \lambda_{0}, 0.5 \lambda_{0}\right),\left(0.35 \lambda_{0}, 0.5 \lambda_{0}\right) ; \quad \# 3\left(0.2 \lambda_{0}, 1.2 \lambda_{0}\right),\left(0.1 \lambda_{0}, 0.2 \lambda_{0}\right)$;

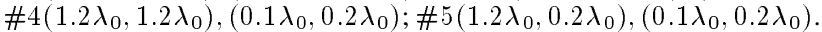

accuracy is not the AIM but the low-order basis functions. Furthermore, the matrix $\overline{\mathbf{Z}}^{\text {near }}$ filling time increases significantly since the number of integration points, in which the Green's function needs to be calculated, is much larger [19].

Finally, the problem is solved with the expansion order $M^{\xi}=$ 3 and the modified AIM expansion (11) on a 256-element mesh with 21168 unknowns. The large average subdomain size $h=$ $2.17 \cdot 10^{-1} \lambda_{0}$ makes an accurate solution with the original AIM expansion (9) next to impossible, as discussed in the previous section. The higher order basis functions combined with the modified expansion (11) allows us to obtain an accurate result as shown in Table I (case 6) and in Fig. 3, in which an excellent agreement between the computed bistatic RCS and the exact Mie series solution is illustrated.

The second example deals with a dielectric cube with the side length $1.4 \lambda_{0}$ illuminated by an $x$-polarized plane wave propagating in the negative $z$ direction. The relative permittivity of the cube is $\varepsilon_{r}=4.0$ and its edges are aligned along the axes of a rectangular $x y z$-coordinate system. To illustrate that the presented AIM technique still maintains the higher order convergence properties of the higher order MoM the RMS error of the bistatic RCS versus the number of unknowns is calculated and plotted in Fig. 4(a) for the expansion orders from $M^{\xi}=1$ to $M^{\xi}=4$. The mesh size is varied from 125 to 42875 hexahedral elements. The AIM grid size $a$ in all simulations is selected as prescribed by the inequality (13b), and the near-field range $d_{\text {near }}$ is chosen large enough to isolate the effect of the expansion order $M^{\xi}$. It is observed that the slope of the convergence curves increases with the expansion order $M^{\xi}$ indicating the higher 
TABLE III

AIM PARAMETERS FOR THE SIMULATIONS IN FIGS. 6 AND 8

\begin{tabular}{l|c|c|c|c|c|c|c|c}
\hline Example & Unknowns & Grid size & $a, \lambda_{0}$ & $b$ (average), $\lambda_{0}$ & $L$ & $d_{\text {near, }} \lambda_{0}$ & Memory AIM & Memory MoM \\
\hline Fig. 6 & 21,600 & $144 \times 144$ & $1.17 \cdot 10^{-1}$ & $2.39 \cdot 10^{-1}$ & $2 \ldots 4$ & $5.0 \cdot 10^{-1}$ & $50 \mathrm{MB}$ & $7 \mathrm{~GB}$ \\
Fig. 8 & 53,574 & $100 \times 100 \times 10$ & $5.84 \cdot 10^{-2}$ & $1.29 \cdot 10^{-1}$ & $2 \ldots 3$ & $3.0 \cdot 10^{-1}$ & $810 \mathrm{MB}$ & $43 \mathrm{~GB}$ \\
\hline
\end{tabular}

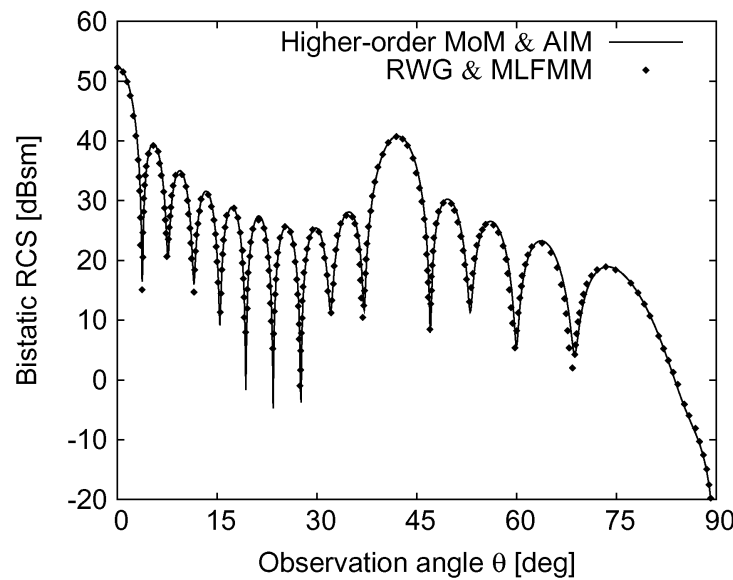

(a)

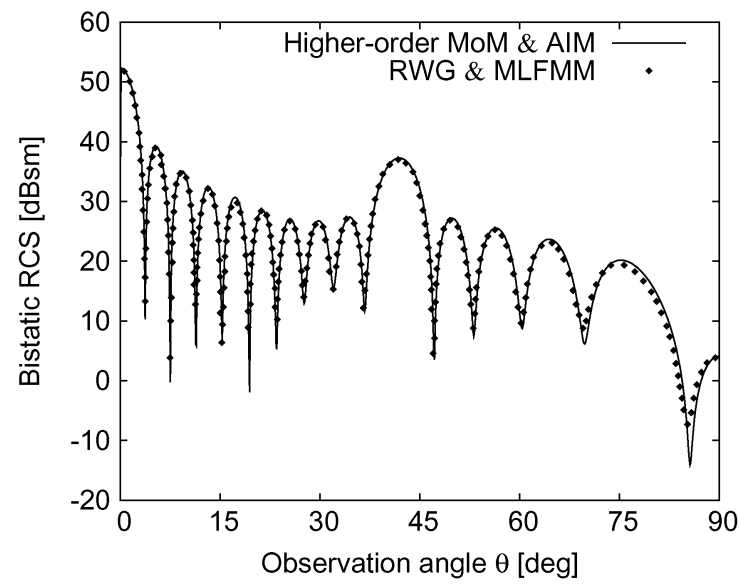

(b)

Fig. 6. Bistatic RCS of the planar $10 \times 10$ ring array. (a) V-V polarization. (b) $\mathrm{H}-\mathrm{H}$ polarization

order convergence of the method. Fig. 4 also shows the computer memory used and the time spent at each iteration versus the number of unknowns. Corresponding values for the classical (unaccelerated) MoM simulations are also given for comparison. As the expansion order $M^{\xi}$ increases the AIM memory usage grows for the same number of unknowns. This is the natural result for the higher order basis functions, since the number of basis functions defined over the same discretization element increases with $M^{\xi}$, which makes the matrix $\overline{\mathbf{Z}}^{\text {near }}$ more populated. However, the computational complexity demonstrates the opposite behavior: The solutions with $M^{\xi}=2$ require less time per iteration than the solutions with $M^{\xi}=1$ and $M^{\xi}=3$. It can be explained as follows. The most of the time spent at each iteration is due to the computation of the matrix-vector product

$$
\overline{\mathbf{Z}} \mathbf{I}=\overline{\mathbf{Z}}^{\text {near }} \mathbf{I}+\overline{\mathbf{Z}}^{\mathrm{far}} \mathbf{I}
$$

TABLE IV

PARAMETERS OF THE AIM, P-FFT AND MLFMM SiMULATIONS OF THE PLANAR $10 \times 10$ RING ARRAY

\begin{tabular}{lcccc}
\hline Technique & Basis functions & Unknowns & Grid size & Memory \\
\hline AIM & $2^{\text {nd }}-3^{\text {rd }}$ order & 21,600 & $144 \times 144$ & $50 \mathrm{MB}^{*}$ \\
P-FFT & $3^{\text {rd } \text { order }}$ & 43,200 & $512 \times 512$ & $109 \mathrm{MB}^{*}$ \\
MLFMM & RWG & 113,400 & - & $331 \mathrm{MB}^{* *}$ \\
\hline
\end{tabular}

* double precision.

** single precision.

where the first and the second terms are calculated by a standard sparse matrix-vector product and the FFT, respectively. As it was stated above, $\overline{\mathbf{Z}}^{\text {near }}$ becomes more dense when the expansion order $M^{\xi}$ increases, and this slows down the computation of the first term in the matrix-vector product (16). At the same time, as it can be seen in Table II, where the parameters of the AIM simulations for selected points in Fig. 4(b) and (c) are given, higher order basis functions are defined in larger hexahedral elements, which allows a coarser AIM auxiliary grid and consequently, the FFT calculation of the second term in the matrix-vector product in (16) is faster. Evidently, for the given example the expansion order $M^{\xi}=2$ provides the best compromise between the density of $\overline{\mathbf{Z}}^{\text {near }}$ and the size of the AIM auxiliary grid. The expansion order $M^{\xi}=3$ is better than $M^{\xi}=1$ and worse than $M^{\xi}=2$ in terms of time spent in each iteration, but the resulting solution is much more accurate as compared to the results for both $M^{\xi}=1$ and $M^{\xi}=2$.

It should be noted that coarser meshes with hexahedral elements larger than those in the example above cannot be utilized since the accuracy condition (10) is violated. Moreover, further increase of the expansion order $M^{\xi}$ leads to unreasonable waste of computational resources both in terms of memory and computational time. Therefore, the use of the basis function expansion orders higher than $M^{\xi}=2$ or $M^{\xi}=3$ is not desirable.

The next example is a planar $10 \times 10$ element array composed of unit cells shown in Fig. 5. The cells are arranged on a square lattice with a period of $1.5 \lambda_{0}$. The geometry is discretized into 2400 third-order quadrilateral elements, and the surface current density is represented up to the order $M^{\xi}=3$, resulting in 21600 unknowns. The bistatic RCS computed for the normally incident plane wave is presented in Fig. 6 along with the reference result from [15] produced by MLFMM [20] with RWG basis functions. The parameters of the AIM simulation are given in Table III.

This array is also studied in [15] by the higher order P-FFT method. Table IV summarizes parameters of the AIM, P-FFT, and MLFMM simulations. The P-FFT and AIM simulations both exploit the planar geometry and solve the problem using the 2-D FFT.

In the final example, AIM for the volume-surface integral equation is tested for a planar $9 \times 9$ array of PEC crosses (Fig. 7) printed on a finite dielectric substrate $\left(\varepsilon_{r}=4.0\right)$. The crosses 


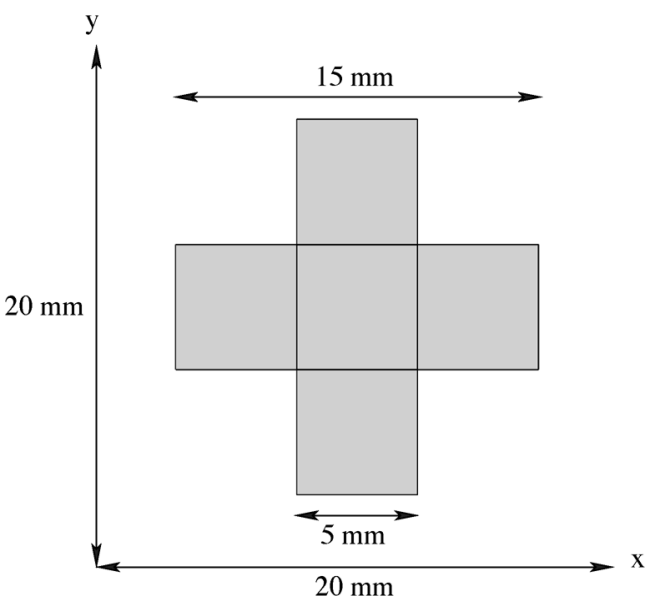

Fig. 7. Unit cell for a $9 \times 9$ array. The PEC crosses are printed on a $180 \times$ $180 \times 1 \mathrm{~mm}^{3}$ dielectric substrate $\left(\varepsilon_{r}=4.0\right)$.

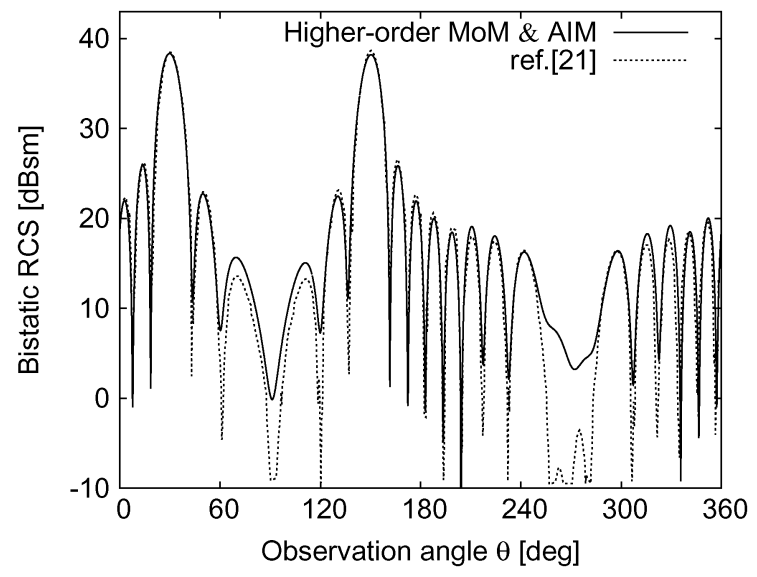

Fig. 8. Bistatic RCS of the $9 \times 9$ array of PEC crosses (Fig. 7) printed on a $180 \times 180 \times 1 \mathrm{~mm}$ dielectric substrate $\left(\varepsilon_{r}=4.0\right)$. The array is illuminated at $9 \mathrm{GHz}$ by a plane wave incident at $\theta=30^{\circ}, \phi=180^{\circ}$. TM polarization.

are arranged on a square lattice with the period $20 \mathrm{~mm}$, and the substrate dimensions are $180 \times 180 \times 1 \mathrm{~mm}^{3}$. The geometry of the problem is meshed with 1369 volume hexahedral and 405 surface quadrilateral elements. The expansion orders $M^{\xi}=3$ and $M^{\xi}=1$ are used for the longitudinal $(x-y)$ and transverse $(z)$ components of the unknown functions, respectively. The total number of unknowns is 53 574. The AIM parameters are summarized in Table III. The bistatic RCS computed at $9 \mathrm{GHz}$ for a plane wave incident from $\theta=30^{\circ}, \phi=180^{\circ}$ is plotted in Fig. 8. The dashed line represents an approximate solution [21], in which an infinite substrate is considered, and the currents on the PEC crosses are assumed to be the same as in the infinite array case. Hence, the largest difference between these two results is observed at grazing angles emphasizing the effects of the substrate truncation.

\section{CONCLUSION}

The AIM for the higher order MoM solution of integral equation is presented. Higher order hierarchical Legendre basis functions are employed to discretize the integral equation. Unlike the classical AIM technique, basis functions that span more than one subdomain are treated independently in each subdomain, thus allowing larger discretization elements and higher basis function expansion orders. Numerical examples for volume, surface and volume-surface integral equations are given to illustrate the performance as well as the higher order convergence of the method.

\section{REFERENCES}

[1] R. F. Harrington, Field Computation by Moment Methods. New York: Wiley-IEEE, 1993.

[2] S. M. Rao, D. R. Wilton, and A. W. Glisson, "Electromagnetic scattering by surfaces of arbitrary shape," IEEE Trans. Antennas Propag., vol. AP-30, no. 3, pp. 409-418, May 1982.

[3] D. H. Schaubert, D. R. Wilton, and A. W. Glisson, "A tetrahedral modeling method for electromagnetic scattering by arbitrarily shaped inhomogeneous dielectric bodies," IEEE Trans. Antennas Propag., vol. 32, no. 1, pp. 77-85, Jan. 1984.

[4] J. M. Song, C. C. Lu, and W. C. Chew, "Multilevel fast multipole algorithm for electromagnetic scattering by large complex objects," IEEE Trans. Antennas Propag., vol. 45, no. 10, pp. 1488-1493, Oct. 1997.

[5] K. Sertel and J. L. Volakis, "Multilevel fast multipole method solution of volume integral equations using parametric geometry modeling," IEEE Trans. Antennas Propag., vol. 52, no. 7, pp. 1686-1692, Jul. 2004.

[6] E. Bleszynski, M. Bleszynski, and T. Jaroszewicz, "AIM: Adaptive integral method for solving large-scale electromagnetic scattering and radiation problems," Radio Science, vol. 31, no. 5, pp. 1225-1251, Sep.-Oct. 1996.

[7] Z. Q. Zhang and Q. H. Liu, "A volume adaptive integral method (VAIM) for 3-D inhomogeneous objects," IEEE Antennas Wireless Propag. Lett., vol. 1, pp. 102-105, 2002.

[8] J. Phillips and J. White, "A precorrected-fft method for electrostatic analysis of complicated 3-d structures," IEEE Trans. Comput.-Aided Design Integr. Circuits Syst., vol. 16, no. 10, pp. 1059-1072, 1997.

[9] X. C. Nie, N. Yuan, L. W. Li, Y. B. Gan, and T. S. Yeo, "A fast volume-surface integral equation solver for scattering from composite conducting-dielectric objects," IEEE Trans. Antennas Propag., vol. 53, no. 2, pp. 818-824, Feb. 2005.

[10] C.-F. Wang, F. Ling, J. Song, and J.-M. Jin, "Adaptive integral solution of combined field integral equation," Microw. Opt. Technol. Lett., vol. 19, no. 5, pp. 321-328, Dec. 1998.

[11] O. S. Kim, P. Meincke, O. Breinbjerg, and E. Jørgensen, "Method of moments solution of volume integral equations using higher-order hierarchical Legendre basis functions," Radio Sci., vol. 39, p. RS5003, 2004, 10.1029/2004RS003041.

[12] K. C. Donepudi, J.-M. Jin, S. Velamparambil, J. Song, and W. C. Chew, "A higher order parallelized multilevel fast multipole algorithm for 3-D scattering," IEEE Trans. Antennas Propag., vol. 49, no. 7, pp. 1069-1078, Jul. 2001.

[13] K. C. Donepudi, J.-M. Jin, and W. C. Chew, "A higher order multilevel fast multipole algorithm for scattering from mixed conducting/dielectric bodies," IEEE Trans. Antennas Propag., vol. 51, no. 10, pp. 2814-2821, Oct. 2003.

[14] M. Meng, J. Hu, and Z. Nie, "On the order and complexity of higher order MLFMA for 3D electromagnetic scattering," in Proc. 3rd Int. Conf. Computat. Electromagn. Its Appl., Nov. 1-4, 2004, pp. 107-110.

[15] S. Gedney, A. Zhu, W. Tang, G. Liu, and P. Petre, "A fast, high-order quadrature sampled pre-corrected fast-Fourier transform for electromagnetic scattering," Microw. Opt. Technol. Lett., vol. 36, no. 5, pp. 343-349, Mar. 2003.

[16] E. Jørgensen, J. L. Volakis, P. Meincke, and O. Breinbjerg, "Higher order hierarchical Legendre basis functions for electromagnetic modeling," IEEE Trans. Antennas Propag., vol. 52, no. 11, pp. 2985-2995, Nov. 2004.

[17] D. S. Sumic and B. M. Kolundžija, "Efficient iterative solution of surface integral equations based on maximaly orthogonalized higher order basis functions," in Proc. 2005 IEEE Antennas Propag. Soc. Int. Symp., Wash., DC, Jul. 2005, vol. 4a, pp. 288-291.

[18] O. S. Kim, P. Meincke, O. Breinbjerg, and E. Jørgensen, "Solution of volume-surface integral equations using higher-order hierarchical Legendre basis functions," Radio Sci., vol. 42, p. RS4023, 2007, 10.1029/ 2006 RS003584.

[19] E. Jørgensen, O. S. Kim, P. Meincke, and O. Breinbjerg, "Higher order hierarchical discretization scheme for surface integral equations for layered media," IEEE Trans. Geosci. Remote Sens., vol. 42, no. 4, pp. 764-772, Apr. 2004. 
[20] J. Song, C. Lu, W. Chew, and S. Lee, "Fast Illinois solver code (FISC)," IEEE Antennas Propag. Mag., vol. 40, no. 3, pp. 27-34, 1998.

[21] J.-M. Jin and J. Volakis, "Electromagnetic scattering by a perfectly conducting patch array on a dielectric slab," IEEE Trans. Antennas Propag., vol. 38, no. 4, pp. 556-563, 1990.

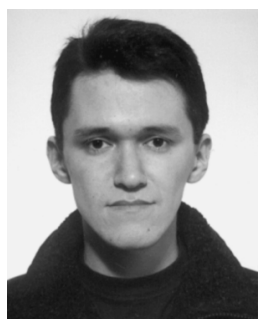

Oleksiy S. Kim received the M.S. and the Ph.D. degree from the National Technical University of Ukraine, Kiev, in 1996 and 2000, respectively, both in electrical engineering. During his Ph.D. studies, his work was primarily concerned with synthesis and optimization of reflector antennas and feed horns, analysis of waveguide structures and discontinuities.

In 2000, he joined the Antenna and Electromagnetics Group, Technical University of Denmark (DTU). He is currently an Associate Professor with the Department of Electrical Engineering, ElectroScience Section, DTU. His current research interests include computational electromagnetics, metamaterials, and modeling of photonic bandgap and plasmonic structures.

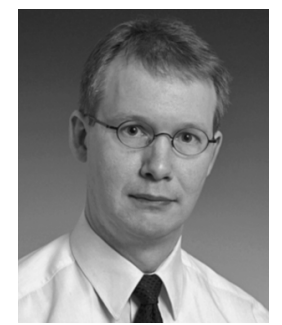

Peter Meincke (S'93-M'96) was born in Roskilde, Denmark, on November 25, 1969. He received the M.Sc.E.E. and Ph.D. degrees from the Technical University of Denmark (DTU), Lyngby, in 1993 and 1996, respectively.

In the spring and summer of 1995 , he was a Visiting Research Scientist with the Electromagnetics Directorate of Rome Laboratory, Hanscom Air Force Base, MA. In 1997, he was with a Danish cellular phone company, working on theoretical aspects of radio-wave propagation. In the spring and summer of 1998, he was visiting the Center for Electromagnetics Research at Northeastern University, Boston, MA, while holding a Postdoctoral position from DTU. In 1999, he became a staff member of the Department of Electromagnetic Systems, DTU. He is currently an Associate Professor with the Department of Electrical Engineering, ElectroScience Section, DTU. His current teaching and research interests include electromagnetic theory and scattering, inverse problems, antenna theory, microwave imaging, and wireless communications.

Dr. Meincke won the first prize award in the 1996 IEEE Antennas and Propagation Society Student Paper Contest in Baltimore, MD, for his paper on uniform physical theory of diffraction equivalent edge currents. Also, he received the 2000 RWP King Paper Award for his paper entitled "Time-domain version of the physical theory of diffraction" published in the February 1999 issue of the IEEE TRANSACTIONS ON ANTENNAS AND PROPAGATION. 